\title{
Non-invasive treatment planning of tandem coronary artery lesions using an interactive planner for PCI
}

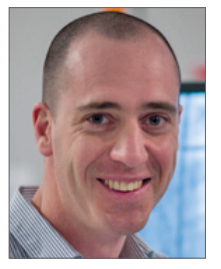

Jeroen Sonck ${ }^{1 *}, \mathrm{MD}$; Yosuke Miyazaki², MD, PhD; Damien Mandry ${ }^{3}, \mathrm{MD}, \mathrm{PhD}$;

Daniele Andreini ${ }^{4}, \mathrm{MD}, \mathrm{PhD}$

1. OLV Clinic Aalst, Aalst, Belgium; 2. Department of Interventional Cardiology, Erasmus Medical Center, Rotterdam, the Netherlands; 3. CHRU Nancy and Université de Lorraine, Nancy, France; 4. Department of Cardiovascular Imaging, Centro Cardiologico Monzino, IRCCS, Milan, Italy

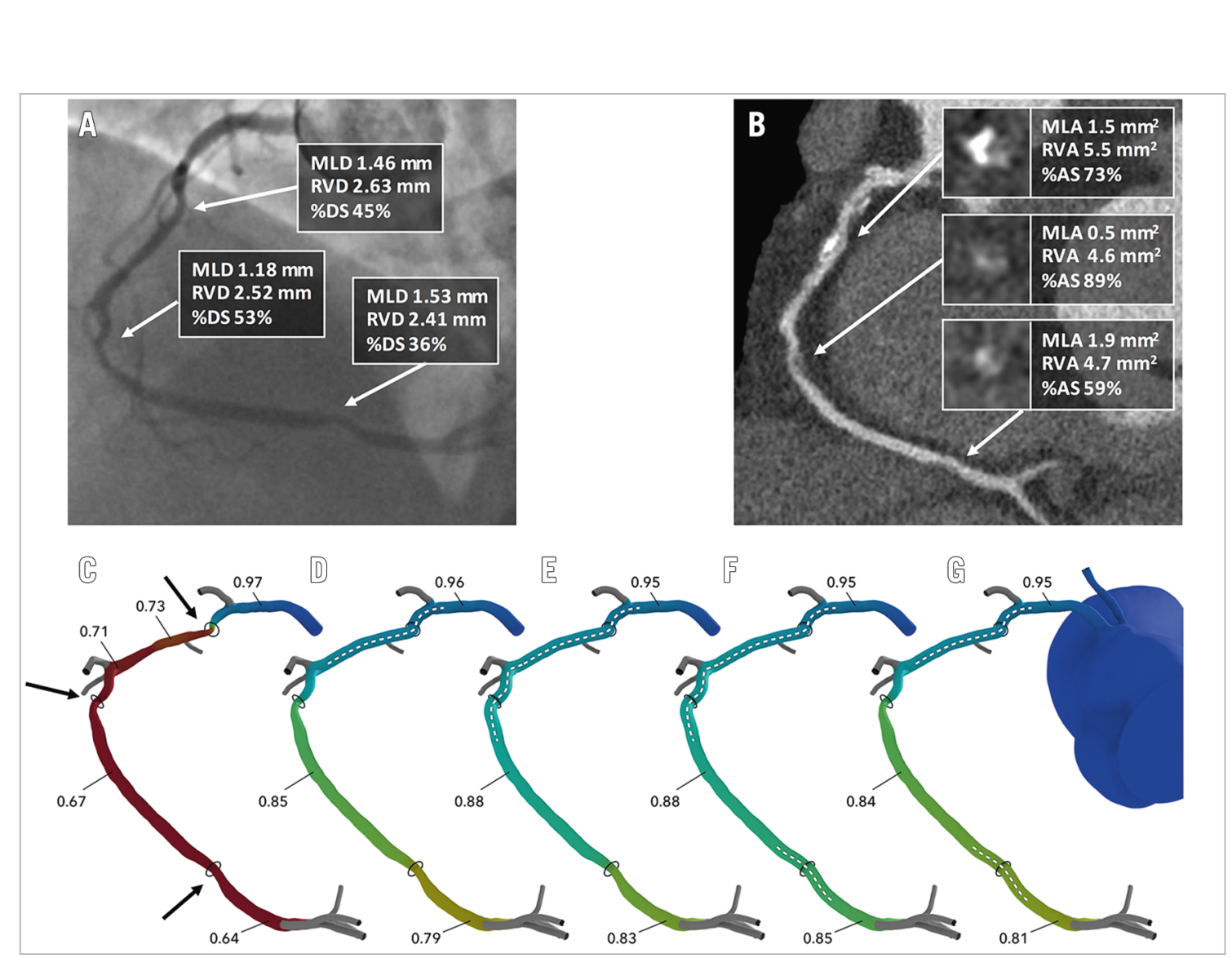

Figure 1. Assessment of PCI planner. A) Coronary angiography of right coronary artery. B) MSCT image of right coronary artery. C) Assessment of FFR ${ }_{C T}$ Target lesions are indicated by arrows. D-G) Assessment of PCI planner for each PCI plan. Stent location marked by dotted line. AS: area stenosis; DS: diameter stenosis; MLA: minimum lumen area; MLD: minimum lumen diameter; MSCT: multislice computed tomography; PCI: percutaneous coronary intervention; RVA: reference vessel area; RVD: reference vessel diameter

*Corresponding author: OLV Clinic Aalst, Moorselbaan 164, B-9300 Aalst, Belgium. E-mail: jeroen.sonck@uzbrussel.be 
Fractional flow reserve derived from coronary computed tomography angiography $\left(\mathrm{FFR}_{\mathrm{CT}}\right)$ is a non-invasive technique providing lesion-specific FFR calculations based on blood flow simulations using patient-specific three-dimensional (3D) geometries ${ }^{1}$. FFR ${ }_{C T}$ has been shown to have a high diagnostic accuracy compared to invasive $\mathrm{FFR}^{2}$. Kim et al previously showed that $\mathrm{FFR}_{\mathrm{CT}}$ could be accurately modelled after updates to the $3 \mathrm{D}$ geometry, but this was an offline process requiring time-consuming manual geometric changes ${ }^{3}$.

The Interactive Planner (HeartFlow, Inc., Redwood City, CA, USA) is computer software enabling prediction of changes in FFR $_{\text {CT }}$ subsequent to virtual removal of coronary artery stenoses. This technology is based on a novel geometric modelling technique to enable physicians to update the luminal geometry efficiently and employ a rapid blood flow solver to compute changes in $\mathrm{FFR}_{\mathrm{CT}}$ in the updated geometry. Changes made to the patient model can be readily visualised and compared alongside the preintervention result.

In the SYNTAX III trial, Heart Teams are randomised to coronary computed tomography angiography (CCTA) or conventional angiography for decision making and treatment planning in stable complex coronary artery disease (CAD), making this an ideal study for gathering data for testing this Interactive Planner ${ }^{4}$.

We present the case of a 52-year-old male patient enrolled in SYNTAX III with a CTA-derived SYNTAX score of 36 and a SYNTAX score II with equipoise risk for surgery or percutaneous coronary intervention (PCI) who presented with serial lesions in the right coronary artery (RCA) (Figure 1A, Figure 1B). The proximal lesion, consisting of a partially calcified plaque, generated an $\mathrm{FFR}_{\mathrm{CT}}$ drop of 0.24 leading to an $\mathrm{FFR}_{\mathrm{CT}}$ of 0.73 . There were two other serial non-calcified lesions in the mid and distal RCA (Figure 1A-Figure 1C).

Four stenting strategies were evaluated (Figure 1D-Figure 1G). In each of the strategies, proximal and distal reference segments were identified and the luminal geometry was restored to match the reference diameters at those locations and transition smoothly in between. Virtual stenting of the proximal lesion alone or in combination with the distal lesion leads to an $\mathrm{FFR}_{\mathrm{CT}}$ of 0.79 and 0.81 , respectively (Figure 1D-Figure 1G). The virtual stenting of the lesions in the proximal and mid RCA (Figure 1E, two stents) or of all three lesions (Figure 1F, three stents) leads to comparable results with predicted $\mathrm{FFR}_{\mathrm{CT}}$ of 0.83 or 0.85 . We recommended the strategy shown in Figure 1E. As demonstrated by this case example, the HeartFlow Interactive Planner technology has the potential to refine procedural strategy and improve patients' outcomes.

\section{Conflict of interest statement}

The authors have no conflicts of interest to declare.

\section{References}

1. Serruys PW, Girasis C, Papadopoulou SL, Onuma Y. Noninvasive fractional flow reserve: scientific basis, methods and perspectives. EuroIntervention. 2012;8:511-9.

2. Nørgaard BL, Leipsic J, Gaur S, Seneviratne S, Ko BS, Ito H, Jensen JM, Mauri L, De Bruyne B, Bezerra H, Osawa K, Marwan M, Naber C, Erglis A, Park SJ, Christiansen EH, Kaltoft A, Lassen JF, Bøtker HE, Achenbach S; NXT Trial Study Group. Diagnostic performance of noninvasive fractional flow reserve derived from coronary computed tomography angiography in suspected coronary artery disease: the NXT trial (Analysis of Coronary Blood Flow Using CT Angiography: Next Steps). J Am Coll Cardiol. 2014;63: $1145-55$.

3. Kim KH, Doh JH, Koo BK, Min JK, Erglis A, Yang HM, Park KW, Lee HY, Kang HJ, Kim YJ, Lee SY, Kim HS. A novel noninvasive technology for treatment planning using virtual coronary stenting and computed tomography-derived computed fractional flow reserve. JACC Cardiovasc Interv. 2014;7:72-8.

4. Cavalcante R, Onuma Y, Sotomi Y, Collet C, Thomsen B, Rogers C, Zeng Y, Tenekecioglu E, Asano T, Miyasaki Y, Abdelghani M, Morel MA, Serruys PW. Non-invasive Heart Team assessment of multivessel coronary disease with coronary computed tomography angiography based on SYNTAX score II treatment recommendations: design and rationale of the randomised SYNTAX III Revolution trial. EuroIntervention. 2017;12: 2001-8. 